(Aus der Kgl. Frauenklinik Dresden [Direktor: Med.-Rat Prof. Dr. E. Kehrer] und aus dem Städt. Säuglingsheim Dresden [Dir. Arzt: Prof. Dr. Rietschel].)

\title{
Hintere Hals- und Nackendrüsen bei Säuglingen in den ersten neun Lebenstagen.
}

\author{
Von \\ Dr. med. Theodor Jensen, \\ Assistenzarzt der Kgl. Frauenklinik.
}

(Eingegangen am 8. Juli 1915.)

Die Rachenmandel wird heute allgemein bei Säuglingen als ein locus minoris resistentiae angesehen. Man weiß durch die Injektionspräparate von Wood, daß die hinteren Hals- und Nackendrüsen - die Glandulae nuchales und die Glandulae poststernomastoideae cervicales - die regionären Lymphdrüsen für die Rachenmandel, ja für das ganze retropharyngeale Gewebe darstellen. Man hat bis jetzt im allgemeinen angenommen, und Czerny erwähnt dies wiederholt in seinen Arbeiten über die exsudative Diathese, daß Nackendrüsen stets ein Beweis dafür sind, daß infektiöse Prozesse im Rachenraum vorhanden sind, ja Czern y sieht in dem Auftreten dieser Drüsen bei jungen Säuglingen ein sicheres Anzeichen einer erhöhten Neigung für Infektionen, einer ,exsudativen Diathese". Indessen fehlen heute noch exakte klinische Beobachtungen in größerem Maßstabe, um mit Sicherheit solche Schlüsse zu ziehen. So ist es z. B. unbekannt, ob auch bei Neugeborenen solch palpable Lymphdrüsen vorkommen, und es fehlt auch uns an einem größeren Material der klinische Beweis, daß Kinder, die solche Nackendrüsen bald nach der Geburt aufweisen, später sicher andere Zeichen der exsudativen Diathese zeigen. Um die eine Lücke hier auszufüllen, habe ich im Jahre 1913 auf Anregung von Herrn Prof. Rietschel-Dresden in der Kgl. Frauenklinik zu Dresden eine größere Anzahl von Kindern auf die erwähnten hinteren Hals- und Nackendrüsen, sowie auf die als Kieferdrüsen bezeichneten Glandulae submaxillares et tonsillares untersucht.

Die Untersuchungen, die gleich nach der Geburt und dann an jedem einzelnen Tage vorgenommen wurden, sind im allgemeinen nicht leicht; es gehört immerhin eine ziemliche Ubung dazu, die Hyperplasie der 
Hals- und Nackendrüsen, sowie der Kieferdrüsen mit Sicherheit zu fühlen. Da jedes Kind ungefähr zehnmal untersucht wurde, so sind im ganzen ca. 2400 Untersuchungen vorgenommen worden. Bei diesen ziemlich zeitraubenden Untersuchungen hat besonders Dr. Römer, seinerzeit Hilfsarzt an der Kgl. Frauenklinik, geholfen, dem ich deshalb bei dieser Arbeit zu Dank verpflichtet bin. Nur bei den Kindern wurde in der folgenden Tabelle ein positiver Ausfall der Untersuchung vermerkt, bei welchen von mir und einem anderen Kollegen Drüsen palpatorisch nachgewiesen wurden. Ein Irrtum, daß es sich wirklich um Drüsen handelt, kann wohl nicht vorliegen. Aus der Tabelle ist zu ersehen, an welchem der 9 Tage, in denen die Kinder in der Regel in der Klinik verbleiben, zum ersten Male und auf welcher Seite (R. oder L.) Drüsen gefunden wurden. In der letzten Rubrik ist neben anderen Angaben über Fieber usw. das Anfangsgewicht der Kinder notiert; K. bedeutet Knabe, M. Mädchen.

\begin{tabular}{|c|c|c|c|c|c|c|c|c|c|c|c|}
\hline Name & 0 & 离 & 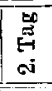 & 㻟 & 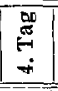 & 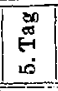 & 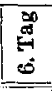 & 蛋 & 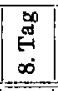 & $\begin{array}{l}\stackrel{5}{\mu} \\
\stackrel{5}{\circ} \\
\end{array}$ & Bemerkungen \\
\hline $\begin{array}{l}\text { 1. Knauth } \\
\text { 22. 9. 12. K. }\end{array}$ & - & L. & L. & L. & L. & L. & L. & L. & L. & L. & $\begin{array}{l}\text { 1. Tag } 3300 \mathrm{~g} \\
\text { 9. Tag } 3050 \mathrm{~g} \\
\text { Fieber }\end{array}$ \\
\hline $\begin{array}{l}\text { 2. Liebscher } \\
\text { 22. 9. 12. K. }\end{array}$ & - & 一 & R. & R. & $\mathbf{R}$. & R. & R. & R. & R. & R. & $\begin{array}{l}\text { 1. Tag } 3100 \mathrm{~g} \\
\text { 9. Tag } 3150 \mathrm{~g}\end{array}$ \\
\hline $\begin{array}{l}\text { 3. Scheringer } \\
\text { 24. 9. 12. K. }\end{array}$ & - & L. & L. & L. & L. & L. & L. & L. & L. & L. & $\begin{array}{l}\text { 1. Tag } 3100 \mathrm{~g} \\
\text { 9. Tag } 3000 \mathrm{~g}\end{array}$ \\
\hline $\begin{array}{l}\text { 4. Fasolt } \\
\text { 18. 9. 12. M. }\end{array}$ & - & L. & L. & L. & L. & L. & L. & L. & L. & L. & $\begin{array}{l}\text { J. Tag } 2600 \mathrm{~g} \\
\text { 9. Tag } 2650 \mathrm{~g}\end{array}$ \\
\hline $\begin{array}{l}\text { 5. Walther } \\
\text { 20.9. 12. M. }\end{array}$ & - & - & - & L. & L. & I. & L. & I. & L. & L. & $\begin{array}{l}\text { 1. Tag } 3200 \mathrm{~g} \\
\text { 9. Tag } 3100 \mathrm{~g}\end{array}$ \\
\hline $\begin{array}{l}\text { 6. Leske } \\
\text { 20.9.12. K. }\end{array}$ & - & - & - & - & - & - & $\mathrm{R}$. & R. & R. & R. & $\begin{array}{l}\text { 1. Tag } 2900 \mathrm{~g} \\
\text { 9. Tag } 2640 \mathrm{~g}\end{array}$ \\
\hline $\begin{array}{l}\text { 7. Rost } \\
\text { 22.9. 12. M. }\end{array}$ & - & 一 & - & - & L. & L. & L. & L. & L. & L. & $\begin{array}{l}\text { 1. Tag } 2300 \mathrm{~g} \\
\text { 9. Tag } 2050 \mathrm{~g} \\
\text { Fieber }\end{array}$ \\
\hline $\begin{array}{l}\text { 8. Hempel } \\
\text { 23.9.12. M. }\end{array}$ & - & R. & R. & R. & R. & R. & R. & $\mathrm{R}$. & R. & R. & $\begin{array}{l}\text { 1. Tag } 2000 \mathrm{~g} \\
\text { 9. Tag } 2020 \mathrm{~g}\end{array}$ \\
\hline $\begin{array}{l}\text { 9. Lehmann } \\
\text { 23.9. 12. K. }\end{array}$ & - & - & - & L. & L. & L. & L. & L. & L. & L. & $\begin{array}{l}\text { 1. Tang } 3300 \mathrm{~g} \\
\text { 9. Tag } 3000 \mathrm{~g} \\
\text { Fieber }\end{array}$ \\
\hline $\begin{array}{l}\text { 10. Kokel } \\
\text { 28.9. 12. M. }\end{array}$ & - & - & - & - & I. & I. & L. & L. & L. & L. & $\begin{array}{l}\text { 1. Tag } 3500 \mathrm{~g} \\
\text { 9. Tag } 3450 \mathrm{~g} \\
\text { Fieber }\end{array}$ \\
\hline $\begin{array}{l}\text { 11. Mosig } \\
\text { 28. 9. 12. M. }\end{array}$ & - & L. & L. & L. & L. & L. & L. & L. & L. & L. & $\begin{array}{l}\text { 1. Tag } 3200 \mathrm{~g} \\
\text { 9. Tag } 3140 \mathrm{~g} \\
12\end{array}$ \\
\hline
\end{tabular}




\begin{tabular}{|c|c|c|c|c|c|c|c|c|c|c|c|}
\hline Name & 0 & $\stackrel{\text { 焉 }}{\stackrel{-}{-}}$ & $\begin{array}{l}\text { 品 } \\
\text { N } \\
\text { N }\end{array}$ & $\underset{\leftrightarrow}{\stackrel{\infty}{\sharp}}$ & $\begin{array}{l}\text { 品 } \\
\stackrel{-1}{+}\end{array}$ & 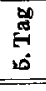 & $\begin{array}{l}\text { 品 } \\
\text { से } \\
0\end{array}$ & 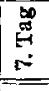 & 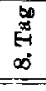 & \begin{tabular}{l}
$\infty$ \\
\multirow{\omega}{*}{} \\
$\sigma^{\circ}$
\end{tabular} & Bemerkungen \\
\hline $\begin{array}{l}\text { 12. Roth } \\
\text { 29. 9. 12. M. }\end{array}$ & - & L. & L. & L. & L. & L. & L. & L. & L. & L. & $\begin{array}{l}\text { 1. Tag } 2400 \mathrm{~g} \\
\text { 9. Tag } 2410 \mathrm{~g}\end{array}$ \\
\hline $\begin{array}{l}\text { 13. Schubert } \\
28.9 .12 . \mathrm{K} \text {. }\end{array}$ & - & - & - & L. & L. & L. & L. & I. & L. & L. & $\begin{array}{l}\text { 1. Tag } 3800 \mathrm{~g} \\
\text { 9. Tag } 3840 \mathrm{~g}\end{array}$ \\
\hline $\begin{array}{l}\text { 14. Wächter } \\
\text { 29.9. 12. M. }\end{array}$ & - & - & - & R. & R. & $R$. & R. & $\mathbf{R}$. & R. & R. & $\begin{array}{l}\text { 1. Tag } 4050 \mathrm{~g} \\
\text { 9. Tag } 3820 \mathrm{~g} \\
\text { Fieber }\end{array}$ \\
\hline
\end{tabular}

15. Kühn

13. 12. 12. $\mathrm{M}$.

16. Polster

2. 5. 13. M.

17. Grüner

3. 5. 13. K.

18. Sehwiel

3. 5. 13. M.

19. Kanneberger

7. 5. 13. M.

20. Heinrich

2. 5. 13. K.

21. Schreiber

7. 5, 13. M.

22. Hoffmann

9. $5.13 . \mathrm{K}$.

23. Hoyer

0. 5. 13. K.

24. Steglich

10. 5. 13. K.

25. Richter

10. 5. 13. M.

26. Rentsch

10. 5. 13. M.

27. Mehnert

10. 5. 13. $M$.

28. Noack

10. 5. 13. K.

29. Reinhardt

14. 5. 13. M.

30. Teschler

15. 5. 13. K.

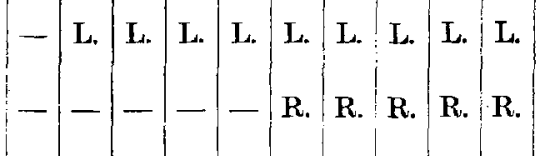

1. Tag $2800 \mathrm{~g}$

9. Tag $2780 \mathrm{~g}$

1. Tag $3770 \mathrm{~g}$

9. Tag $3630 \mathrm{~g}$

Angina der Mutter am 3. Tage.

I. Tag $2050 \mathrm{~g}$

9. Tag $2120 \mathrm{~g}$

1. Tag $2900 \mathrm{~g}$

9. Tag $3060 \mathrm{~g}$

1. Tag $4600 \mathrm{~g}$

9. Tag $4550 \mathrm{~g}$

1. Tag $3200 \mathrm{~g}$

9. Tag $3300 \mathrm{~g}$

1. Tag $2900 \mathrm{~g}$

9. Tag $3060 \mathrm{~g}$

1. Tag $3500 \mathrm{~g}$

9. Tag $3520 \mathrm{~g}$

1. Tag $3450 \mathrm{~g}$

9. Tag $3570 \mathrm{~g}$

1. Tag $2800 \mathrm{~g}$

9. Tag $2650 \mathrm{~g}$

Fieber

1. Tag $1900 \mathrm{~g}$

9. Tag $1930 \mathrm{~g}$

1. Tag $3200 \mathrm{~g}$

9. $\mathrm{Tag} 3070 \mathrm{~g}$

Fieber

1. Tag $3500 \mathrm{~g}$

9. Tag $3700 \mathrm{~g}$

1. Tag $2450 \mathrm{~g}$

9. Tag $2520 \mathrm{~g}$

1. Tag $2500 \mathrm{~g}$

9. Tag $2510 \mathrm{~g}$

1. Tag $3000 \mathrm{~g}$

9. Tag $3020 \mathrm{~g}$ 


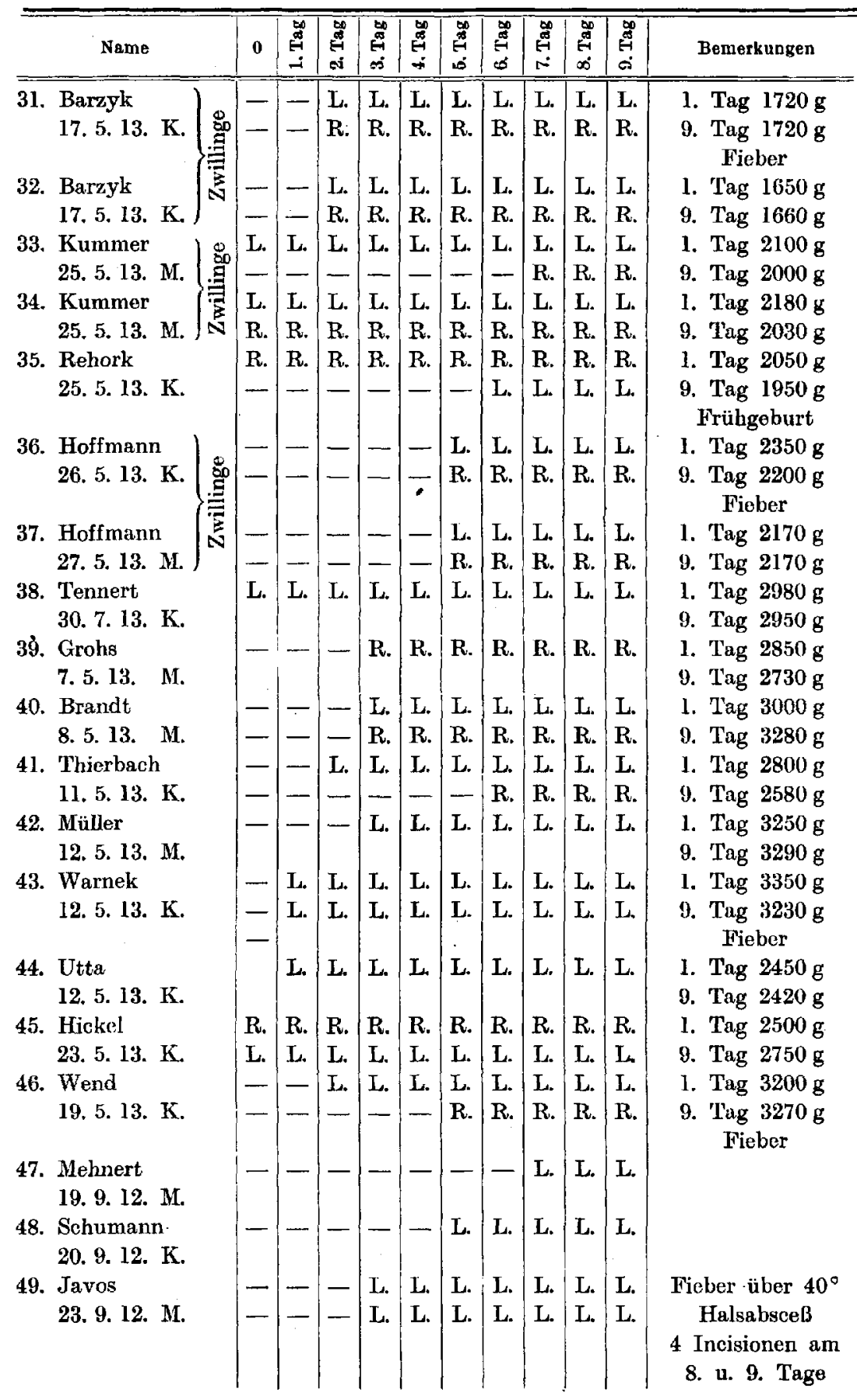




\begin{tabular}{|c|c|c|c|c|c|c|c|c|c|c|c|c|}
\hline & Name & 0 & 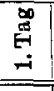 & 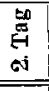 & 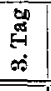 & $\begin{array}{l}0 \\
\stackrel{\infty}{6} \\
\stackrel{4}{+} \\
+\end{array}$ & is & \begin{tabular}{l}
0 \\
\multirow{3}{*}{} \\
0 \\
0
\end{tabular} & 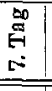 & 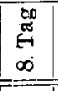 & 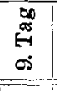 & Bemerkungen \\
\hline 50. & $\begin{array}{l}\text { Lose } \\
\text { 25. 9. 13. M. }\end{array}$ & - & 一 & - & - & - & -1 & L. & L. & L. & L. & 3250 \\
\hline & $\begin{array}{l}\text { Triebisch } \\
\text { 26. 9. 13. K. }\end{array}$ & - & - & - & 一 & - & - & L. & I. & L. & L. & 2300 \\
\hline $52:$ & $\begin{array}{l}\text { Friebel } \\
26.9 .13 . \mathrm{K} .\end{array}$ & - & - & - & R. & R. & $\mathrm{R}$. & R. & R. & R. & R. & 1. Tag $2900 \mathrm{~g}$ \\
\hline & $\begin{array}{l}\text { Schuster } \\
29.9 .13 . \text { M. }\end{array}$ & - & 一 & - & I. & L. & I. & L. & L. & L. & L. & 1. Tag $3250 \mathrm{~g}$ \\
\hline & $\begin{array}{l}\text { Schneider } \\
\text { 21. 9. 13, K. }\end{array}$ & - & $\mathrm{R}$. & R. & R. & R. & R. & R. & R. & R. & R. & 1. Tag $2710 \mathrm{~g}$ \\
\hline 55. & $\begin{array}{l}\text { Winkler. } \\
\text { 2. 10. 12. M. }\end{array}$ & - & R. & R. & R. & R. & R. & R. & R. & $\mathbf{R}$. & R. . & 1. $\mathrm{Tag} 2500 \mathrm{~g}$ \\
\hline 56. & $\begin{array}{l}\text { Brüner } \\
\text { 13. 10. 12. M. }\end{array}$ & - & $\mathrm{R}$. & $\mathrm{R}$. & R. & R. & $\mathrm{R}$. & R. & $\mathrm{I}$. & R. & R. & 1. Tag $3200 \mathrm{~g}$ \\
\hline 57. & $\begin{array}{l}\text { Brenckel } \\
\text { 25. 9. 12. K. }\end{array}$ & - & R. & R. & R. & R. & R. & R. & $\mathrm{R}$. & R. & R. & 1. 'Tag $1700 \mathrm{~g}$ \\
\hline 58. & $\begin{array}{l}\text { Becker } \\
\text { 26.9. 13. K. }\end{array}$ & 一 & L. & L. & La & L. & L. & L. & L. & L. & L. & 1. 'Tug $3250 \mathrm{~g}$ \\
\hline 59. & $\begin{array}{l}\text { Arend } \\
30.9 .12 . \mathrm{K} .\end{array}$ & L. & L. & L. & L. & L. & L. & L. & L. & L. & L. & $\begin{array}{l}\text { Kaiserschnitt } \\
\text { 1. Tag } 3150 \mathrm{~g}\end{array}$ \\
\hline 60. & $\begin{array}{l}\text { Fleischer } \\
\text { 4. 10. 12. K. }\end{array}$ & - & R. & R. & R. & R. & R. & R. & R. & R. & R. & 1. Tag $2900 \mathrm{~g}$ \\
\hline & $\begin{array}{l}\text { Rudolf } \\
\text { 2.5.13. K. }\end{array}$ & - & - & - & - & - & - & L. & L. & L. & L. & 1. Tag $3450 \mathrm{~g}$ \\
\hline & Höhnemann & - & R. & R. & R. & R. & R. & R. & R. & R. & R. & 1. 'Iag $3250 \mathrm{~g}$ \\
\hline & $\begin{array}{l}\text { 7. 5. 13. K. } \\
\text { Müller }\end{array}$ & - & - & $\overline{\mathrm{R}}$. & $\overline{\mathrm{R}}$. & $\begin{array}{l}\text { L. } \\
\text { R. }\end{array}$ & $\begin{array}{l}\text { L. } \\
\text { R. }\end{array}$ & $\begin{array}{l}\text { L. } \\
\text { R. }\end{array}$ & $\begin{array}{l}\text { L. } \\
\text { R. }\end{array}$ & $\begin{array}{l}\text { L. } \\
\text { R. }\end{array}$ & L. & 1. Tag $3250 \mathrm{~g}$ \\
\hline & 8.5.13. M. & - & - & - & - & L. & L. & L. & L. & L. & T. & \\
\hline 64. & $\begin{array}{l}\text { Frauendorf } \\
\text { 16. 5. 13. M. }\end{array}$ & - & - & - & - & - & R. & R. & R. & R. & R. & 1. 'lang $2500 \mathrm{~g}$ \\
\hline 65. & $\begin{array}{l}\text { Hauptmann } \\
\text { 16. 5. 13. M. }\end{array}$ & - & - & L. & L. & L. & L. & L. & L. & L. & L. & 1. Tag $2500 \mathrm{~g}$ \\
\hline 60. & $\begin{array}{l}\text { Pech } \\
\text { 15. 5. 13. }\end{array}$ & - & - & - & - & R. & R. & $\begin{array}{l}\text { R. } \\
\text { L. }\end{array}$ & $\begin{array}{l}\text { R. } \\
\text { L. }\end{array}$ & $\begin{array}{l}\text { R. } \\
\text { L. }\end{array}$ & $\begin{array}{l}\text { R. } \\
\text { L. }\end{array}$ & 1. Tang $3450 \mathrm{~g}$ \\
\hline & $\begin{array}{l}\text { Jäger } \\
\text { 19. 5. 13. K. }\end{array}$ & $\begin{array}{l}\text { R. } \\
\text { L. }\end{array}$ & $\begin{array}{l}\text { R. } \\
\text { L. }\end{array}$ & $\begin{array}{l}\text { R. } \\
\text { L. }\end{array}$ & $\begin{array}{l}\text { R. } \\
\text { L. }\end{array}$ & $\begin{array}{l}\text { R. } \\
\text { L. }\end{array}$ & $\begin{array}{l}\text { R. } \\
\text { C. }\end{array}$ & $\begin{array}{l}\text { R. } \\
\text { L. }\end{array}$ & R. & $\begin{array}{l}\text { R. } \\
\text { L. }\end{array}$ & $\begin{array}{l}\text { R. } \\
\text { L. }\end{array}$ & 1. Tag $3250 \mathrm{~g}$ \\
\hline 68. & $\begin{array}{l}\text { Riegel } \\
\text { 18. 5. 13. K. }\end{array}$ & - & - & - & $\begin{array}{l}\text { R. } \\
\text { L. }\end{array}$ & $\begin{array}{l}\mathrm{R} . \\
\mathrm{L} .\end{array}$ & $\begin{array}{l}\text { R. } \\
\text { L. }\end{array}$ & $\begin{array}{l}\text { R. } \\
\text { L. }\end{array}$ & $\begin{array}{l}\text { R. } \\
\text { L. }\end{array}$ & $\begin{array}{l}\mathrm{R} . \\
\mathrm{L} .\end{array}$ & $\begin{array}{l}\text { R. } \\
\text { L. }\end{array}$ & 1. Iag $3400 \mathrm{~g}$ \\
\hline & $\begin{array}{l}\text { Graschwitz } \\
\text { 19.5. 13. M. }\end{array}$ & - & - & $\mathrm{L}$. & $\mathrm{L}$. & L. & $\mathrm{I}$. & L. & L. & L. & $\mathrm{L}$. & $\begin{array}{l}\text { 1. Tag } 3000 \mathrm{~g} \\
\text { Stcißlage }\end{array}$ \\
\hline 70. & $\begin{array}{l}\text { Kirsten } \\
\text { 19. 5. 13. M. }\end{array}$ & 一 & - & - & - & - & - & - & L. & I. & L. & 1. Tag $2700 \mathrm{~g}$ \\
\hline 71. & $\begin{array}{l}\text { Schneckau } \\
\text { 21. 5. 13. K. }\end{array}$ & - & - & -1 & - & - & $\begin{array}{l}\text { R. } \\
\text { L. }\end{array}$ & $\begin{array}{l}\text { R. } \\
\text { L. }\end{array}$ & $\begin{array}{l}\text { R. } \\
\text { I. }\end{array}$ & $\begin{array}{l}\text { R. } \\
\text { L. }\end{array}$ & $\begin{array}{l}\text { R. } \\
\text { L. }\end{array}$ & 1. Tag $3100 \mathrm{~g}$ \\
\hline
\end{tabular}




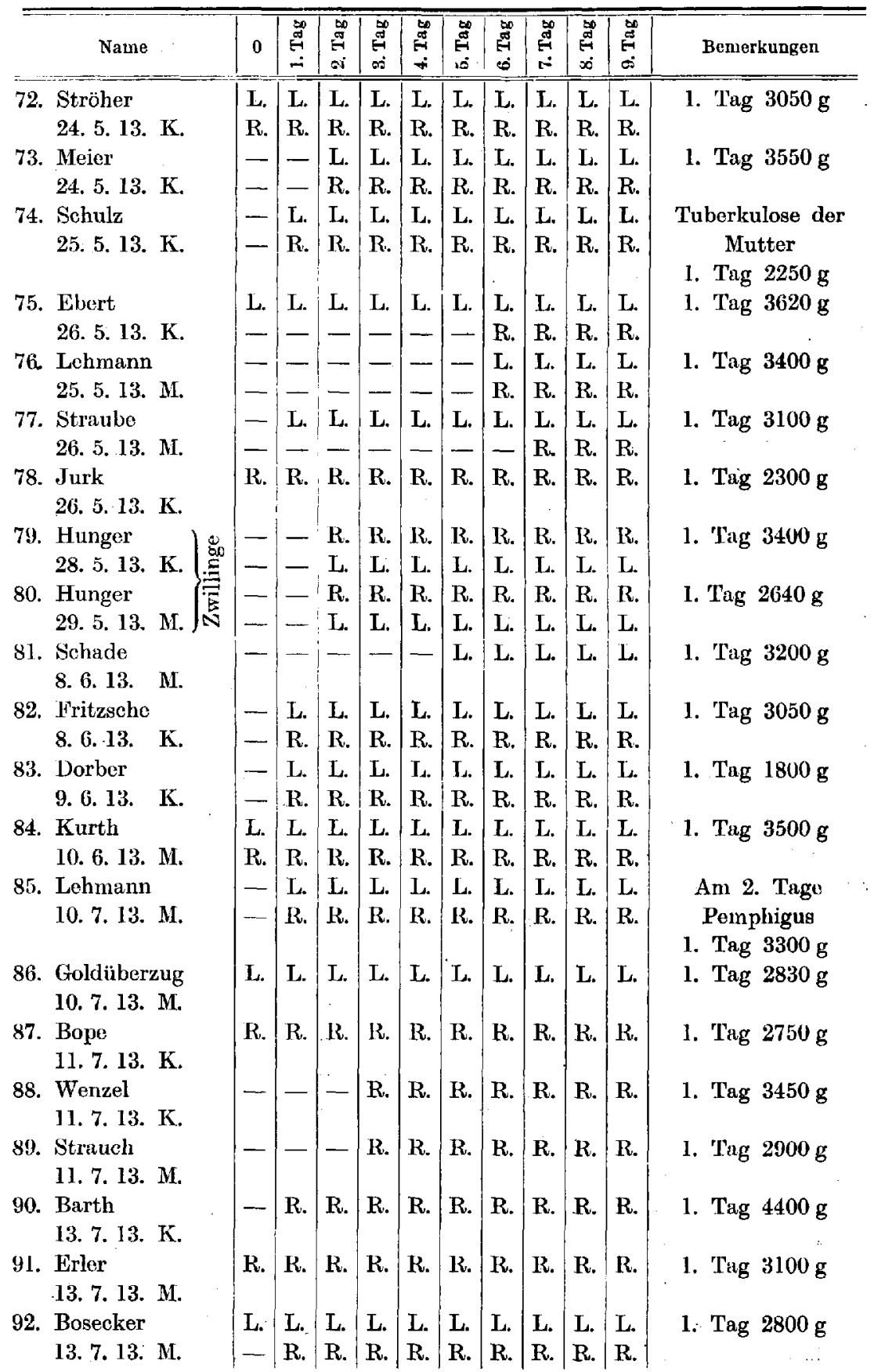




\begin{tabular}{|c|c|c|c|c|c|c|c|c|c|c|c|}
\hline Name & 0 & ผี & 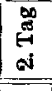 & $\underset{\omega}{\infty}$ & $\underset{+}{\stackrel{\oplus}{*}+\infty}$ & 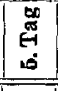 & 总 & 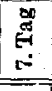 & 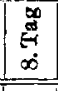 & $\begin{array}{l}\infty \\
\stackrel{\infty}{\oplus} \\
\infty \\
\end{array}$ & Bemerkungen \\
\hline 93. Weidemann & - & - & - & - & - & $\mathbf{R}$. & R. & R. & R. & $\mathbf{R}$. & 1. Tag $2600 \mathrm{~g}$ \\
\hline 13. 7. 13. K. & - & - & - & - & - & L. & L. & L. & L. & L. & \\
\hline 94. Tschorm & $R$. & $R$. & $R$. & $R$. & $\mathbf{R}$. & R. & $\mathbf{R}$. & $R$. & R. & $\mathbf{R}$. & 1. Tag $3300 \mathrm{~g}$ \\
\hline 14. 7. 13. M. & L. & L. & L. & L. & $\mathrm{L}$. & L. & L. & L. & L. & L. & \\
\hline $\begin{array}{l}\text { 95. Lange } \\
\text { 15. } 7.13 . \mathrm{K} \text {. }\end{array}$ & - & - & L. & L. & L. & L. & L. & L. & L. & L. & 1. Tag $2800 \mathrm{~g}$ \\
\hline $\begin{array}{l}\text { 96. Thierbach } \\
\text { 6. 7. } 13 . \mathrm{K} \text {. }\end{array}$ & 一 & - & - & R. & $\mathrm{R}$. & R. & $R$. & R. & R. & $\mathrm{R}$. & 1. Tag $3250 \mathrm{~g}$ \\
\hline $\begin{array}{l}\text { 97. Prüfer } \\
\text { 16. 7. 13. M. }\end{array}$ & - & - & - & R. & R. & R. & $R$. & R. & R. & R. & 1. Tag $3200 \mathrm{~g}$ \\
\hline $\begin{array}{l}\text { 98. Schrieger } \\
\text { 19. 7. 13. M. }\end{array}$ & 一 & - & - & - & - & - & L. & L. & L. & L. & 1. Tag $3800 \mathrm{~g}$ \\
\hline $\begin{array}{l}\text { 99. Brandstadt } \\
\text { 19. 7. 13. M. }\end{array}$ & $\begin{array}{l}\text { R. } \\
\text { L. }\end{array}$ & $\begin{array}{l}\text { R. } \\
\text { L. }\end{array}$ & $\begin{array}{l}\text { R. } \\
\text { L. }\end{array}$ & $\begin{array}{l}\text { R. } \\
\text { L. }\end{array}$ & $\begin{array}{l}\text { R. } \\
\text { L. }\end{array}$ & $\begin{array}{l}\text { R. } \\
\text { L. }\end{array}$ & $\begin{array}{l}\text { R. } \\
\text { L. }\end{array}$ & $\begin{array}{l}\text { R. } \\
\text { L. }\end{array}$ & $\begin{array}{l}\text { R. } \\
\text { L. }\end{array}$ & $\begin{array}{l}\text { R. } \\
\text { L. }\end{array}$ & 1. Tug $3350 \mathrm{~g}$ \\
\hline $\begin{array}{l}\text { 100. Strohmeyer } \\
\text { 19. 7. 13. M. }\end{array}$ & - & - & L. & L. & L. & L. & L. & L. & L. & L. & 1. $\operatorname{Tag} 3250 \mathrm{~g}$ \\
\hline $\begin{array}{l}\text { 101. Schröder } \\
\text { 19. 7. 13. M. }\end{array}$ & - & - & R. & R. & R. & R. & $\mathrm{R}$. & R. & R. & R. & 1. $\operatorname{Tag} 2250 \mathrm{~g}$ \\
\hline $\begin{array}{l}\text { 102. Bode } \\
\text { 20.7. 13. M. }\end{array}$ & $\begin{array}{l}\text { L. } \\
\text { R. }\end{array}$ & $\begin{array}{l}\text { L. } \\
\text { R. }\end{array}$ & $\begin{array}{l}\text { L. } \\
\text { R. }\end{array}$ & $\begin{array}{l}\mathrm{L} . \\
\mathrm{R} .\end{array}$ & $\begin{array}{l}\text { L. } \\
\text { R. }\end{array}$ & $\begin{array}{l}\text { L. } \\
\text { R. }\end{array}$ & $\begin{array}{l}\text { L. } \\
\text { R. }\end{array}$ & $\begin{array}{l}\text { L. } \\
\text { R. }\end{array}$ & $\begin{array}{l}\text { L. } \\
\mathrm{R} \text {. }\end{array}$ & $\begin{array}{l}\text { L. } \\
\text { R. }\end{array}$ & 1. Tag $3500 \mathrm{~g}$ \\
\hline $\begin{array}{l}\text { 103. Müller } \\
\text { 16. 5. 13. M. }\end{array}$ & - & - & - & - & - & R. & R. & R. & R. & R. & 1. Tag $3100 \mathrm{~g}$ \\
\hline $\begin{array}{l}\text { 104. Lehmann } \\
11,5,13 . \mathrm{M} \text {. }\end{array}$ & -1 & - & - & - & - & L. & $\begin{array}{l}\mathrm{L} . \\
\mathrm{R} \text {. }\end{array}$ & $\begin{array}{l}\text { L. } \\
\text { R. }\end{array}$ & $\left|\begin{array}{l}\text { L. } \\
\text { R. }\end{array}\right|$ & $\begin{array}{l}\mathrm{L} . \\
\mathrm{R} .\end{array}$ & 1. 'Tag $2800 \mathrm{~g}$ \\
\hline
\end{tabular}

Im ganzen kamen 240 Kinder zur Untersuchung. Bei 104, das sind ca. $43 \%$, von ihnen waren, wie aus der Tabelle hervorgeht, Nackendrüsen nachweisbar. Die Nackendrüsen wurden nachgewiesen

24 mal gleich nach der Geburt, $24 \mathrm{mal}$ vom 1. Tage an,

$\begin{array}{rllll}16 \mathrm{mal} & , & 2 . & , & , \\ 14 \mathrm{mal} & , & 3 . & , & , \\ 5 \mathrm{mal} & , & 4 . & , & , \\ 9 \mathrm{mal} & , & 5 . & , & , \\ 4 \mathrm{mal} & , & 6 . & , & , \\ 2 \mathrm{mal} & , & 7 . & , & ,\end{array}$

46 mal wurden die Kinder 3 stündlich gemessen und bei 12 von diesen zeigte sich Fieber; die anderen waren in den ersten neun Tagen fieberfrei. Irgendein direkter Zusammenhang des Fiebers mit den Lymphdrüsenschwellungen konnte nicht nachgewiesen werden. Von diesen 46 Kindern erreichten 23 nicht das Initialgewicht am 9. Tage. 
Geschwollene Kieferdrüsen, welche durch Lymphgefäßstränge mit den Tonsillen in Verbindung stehen, habe ich bei den Kindern in den ersten neun Lebenstagen nicht ein einziges Mal nachweisen können, allerdings sind sie nicht so leicht der Palpation zugänglich.

Welche Schlüsse dürfen wir nun aus unseren Beobachtungen ziehen? Wir meinen, daB Lymphdrüsenschwellungen der Nackengegend beim Kind in den ersten Lebenstagen durchaus nicht immer durch exogene Infektionen vom Rachenraum bedingt sein müssen, wie es z. B. Czerny annimmt. Man mag zugeben, daß bei einem Teil dieser Kinder eine Infektion im Spiele ist, und man kann sich sehr wohl vorstellen, daß die Drüsenschwellungen mit dem ersten Eintritt von Scheidenbakterien in den Mund des Kindes bei der Passage durch den Geburtskanal zusammenhängen können, oder daß eine leichte Infektion des adenoiden Gewebes stattfindet (Schnupfen, Grippe). Doch muß es unbedingt noch andere Ursachen für die Drüsenhyperplasien geben, da sie in einem unserer Fälle bei einem Kind nachgewiesen wurden, das durch klassischen Kaiserschnitt, der vor erfolgtem Blasensprung und vor Eintritt des kindlichen Kopfes ins Becken ausgeführt wurde, zur Welt kam und da sodann die häufigen Befunde von Drüsenschwellungen gleich nach der Geburt unmöglich durch eine Infektion erklärt werden können.

! Sodann glauben wir berechtigt zu sein, bei der großen Häufigkeit des Befundes diesem keine allzu große pathologische Bedeutung zusprechen zu dürfen. Es scheint uns auch zweifelhaft, ob die Drüsenhyperplasien stets mit einer angeborenen Konstitutionsanomalie - anch bei Ausschluß einer Infektion - in Beziehung zu bringen sind. Wir können bis jetzt nur das mit Sicherheit sagen, daß das Kind der ersten Lebenstage sehr leicht zu Lymphdrüsenschwellungen, besonders zu solchen der gut palpablen Nackendrüsen neigt, und daß diese Schwellungen nicht nur angeboren sein können, sondern sogar bei Kindern beobachtet werden, die den Geburtskanal nicht passiert haben, vielmehr durch Kaiserschnitt zur Welt gekommen sind (wie Fall Nr. 59 lehrt).

Ob Kinder mit Drüsenhyperplasien, also sog. „Drüsenkinder", später Lymphatiker im Sinne He ubners oder gar exsudativ im Sinne Czernys werden, darüber läßt sich bis jetzt nichts Sicheres aussagen; erst Jahre hindurch konsequent geführte Aufzeichnungen können bei größerem Material diese Frage entscheiden.

Nachtrag: Durch meine Einberufung ist das Erscheinen der Arbeit verzögert worden. So ist es gekommen, daß sich bereits Coerper 
184 Th. Jensen: Hintere Hals- und Nackendrüsen bei Säuglingen usw.

(Monatsschr. f. Kinderheilk. Bd. XIII, S. 462) mit meiner Arbeit befaßt, die ihm durch den Korrekturbogen bekannt war. Coerper hat nun bei Neugeborenen in den ersten Lebenstagen niemals die hinteren Hals- und Nackendrüsen palpieren können, wohl aber bei fast allen Neugeborenen Achseldrüsen. Erst vom 3. Tage sind nach ihm auch Hals- und Nackendrüsen palpabel, und Coerper will deshalb diese Vergrößerung ,stets" auf infektiöse Prozesse zurückführen. Wir halten unsere Befunde voll aufrecht, und müssen auch ablehnen, daß dafür Täuschungen in der Untersuchung verantwortlich gemacht werden sollen. Gerade auf die Technik haben wir besonders geachtet und wenn die Tatsache, die auch Coerper zugibt, feststeht, da $B$ es angeborene palpable Lymphdrüsen beim Neugeborenen gibt, ja dies ein sehr häufiges Symptom z. B. für die Achseldrüsen ist, so sehen wir keinen Grund ein, diese Tatsache für andere Lymphdrüsen nicht anerkennen zu wollen. 


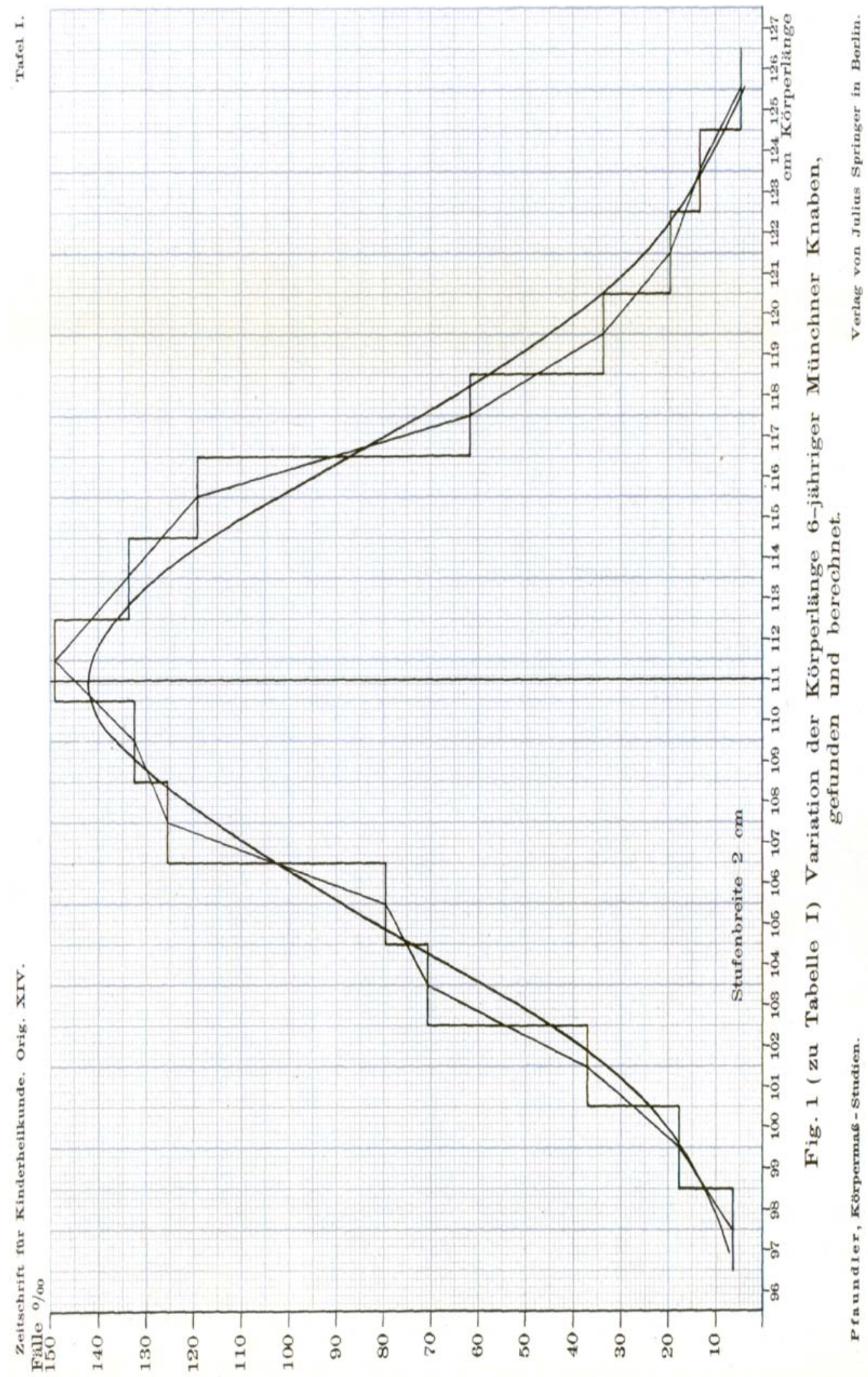


80 焉

EE⿱

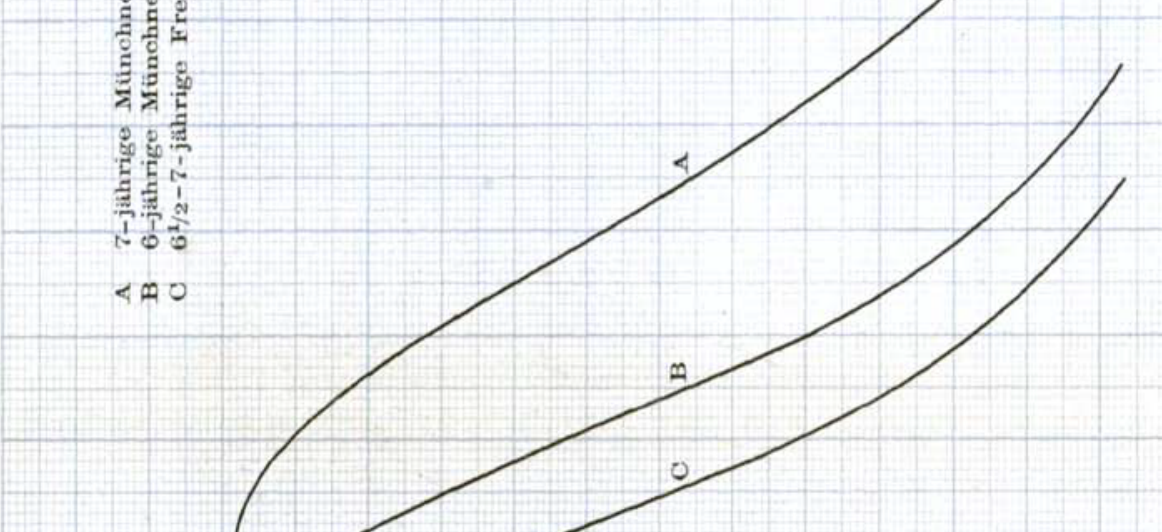

400

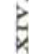
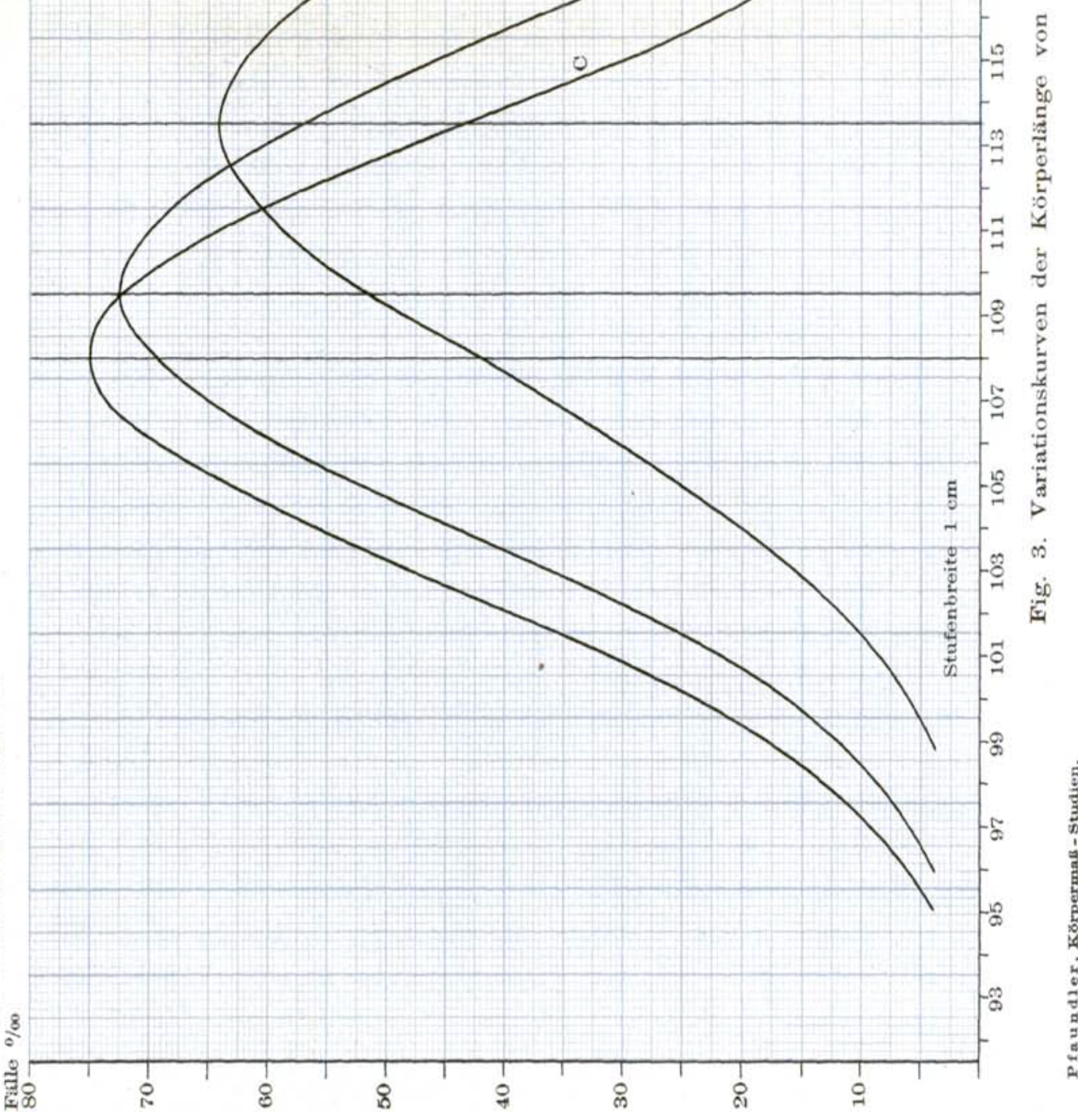
굴

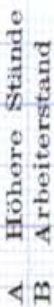

两

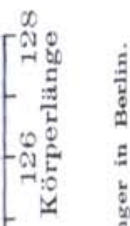

तो है

$-$

$\frac{-1}{-9}$

일

$-3$

$\stackrel{\infty}{=}$

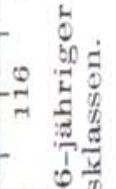

$\rightarrow \quad 00$

$-80$

营要

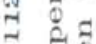

- के

- 10

त

(

$\infty$

$\approx ?$

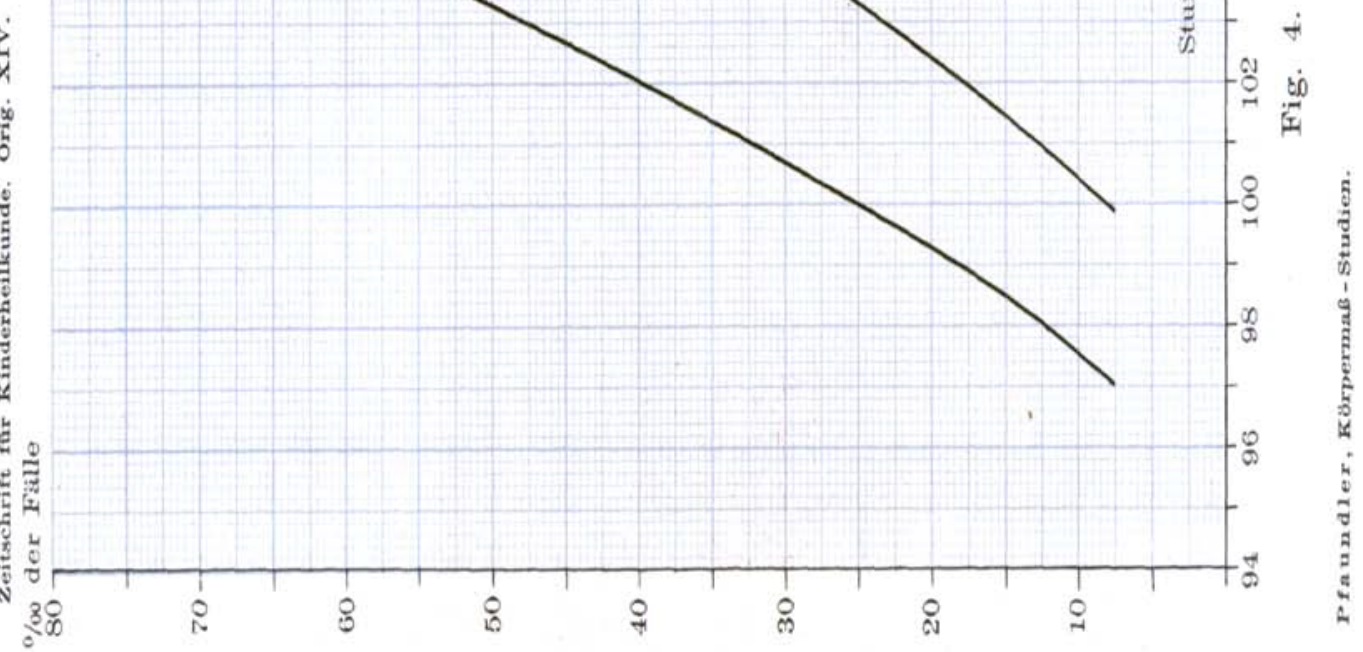




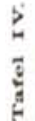

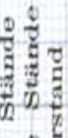

2.

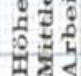

$\triangle 40$

रे

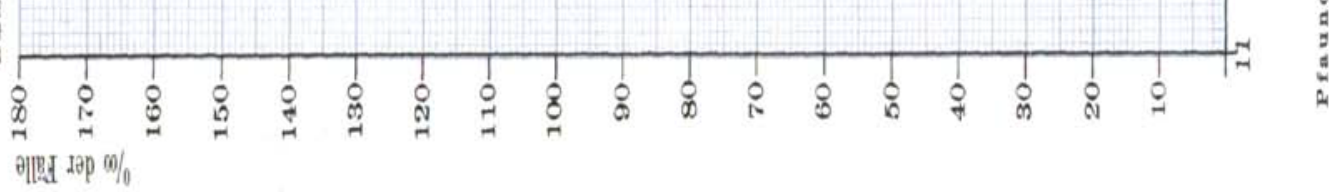




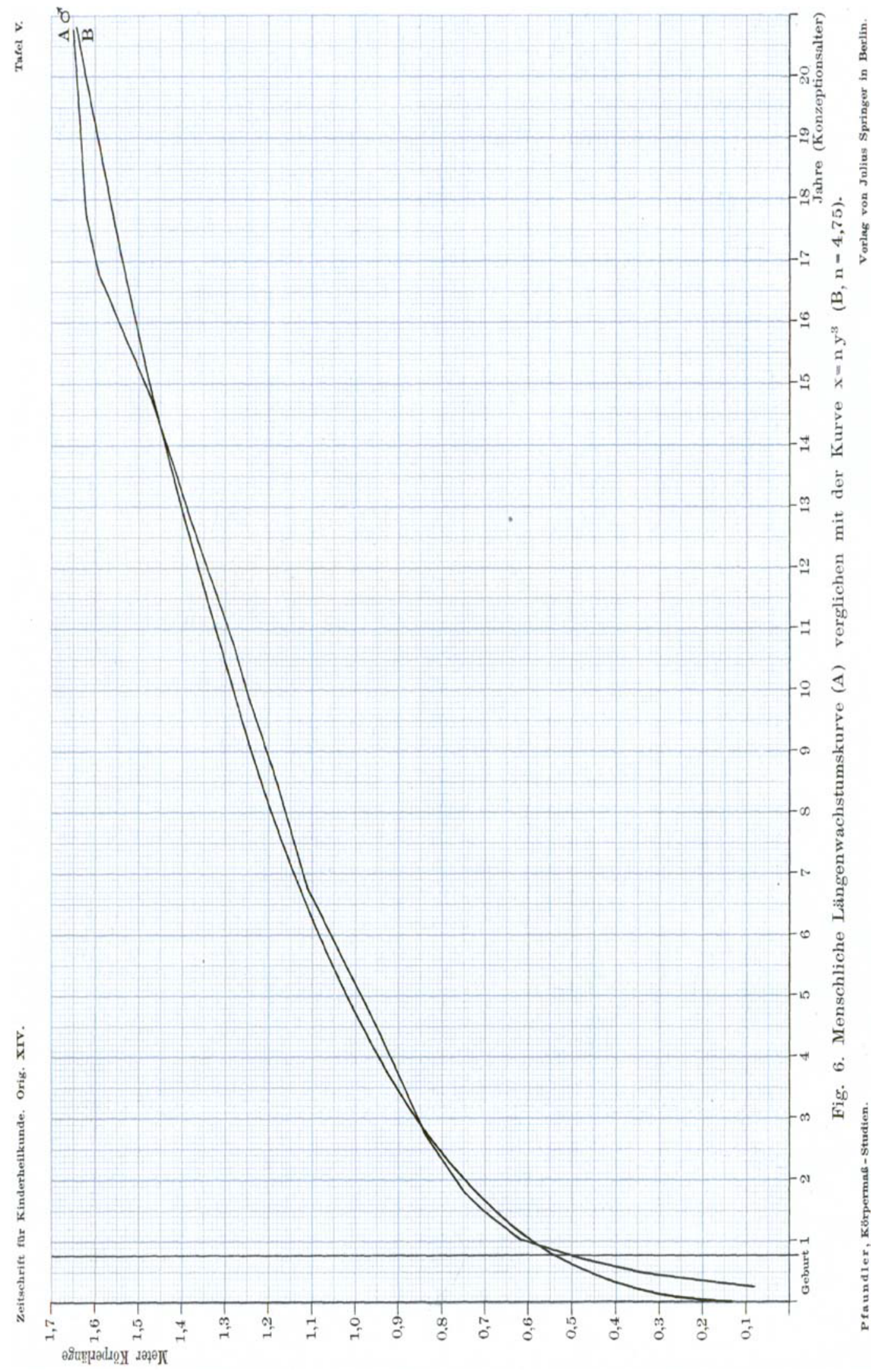




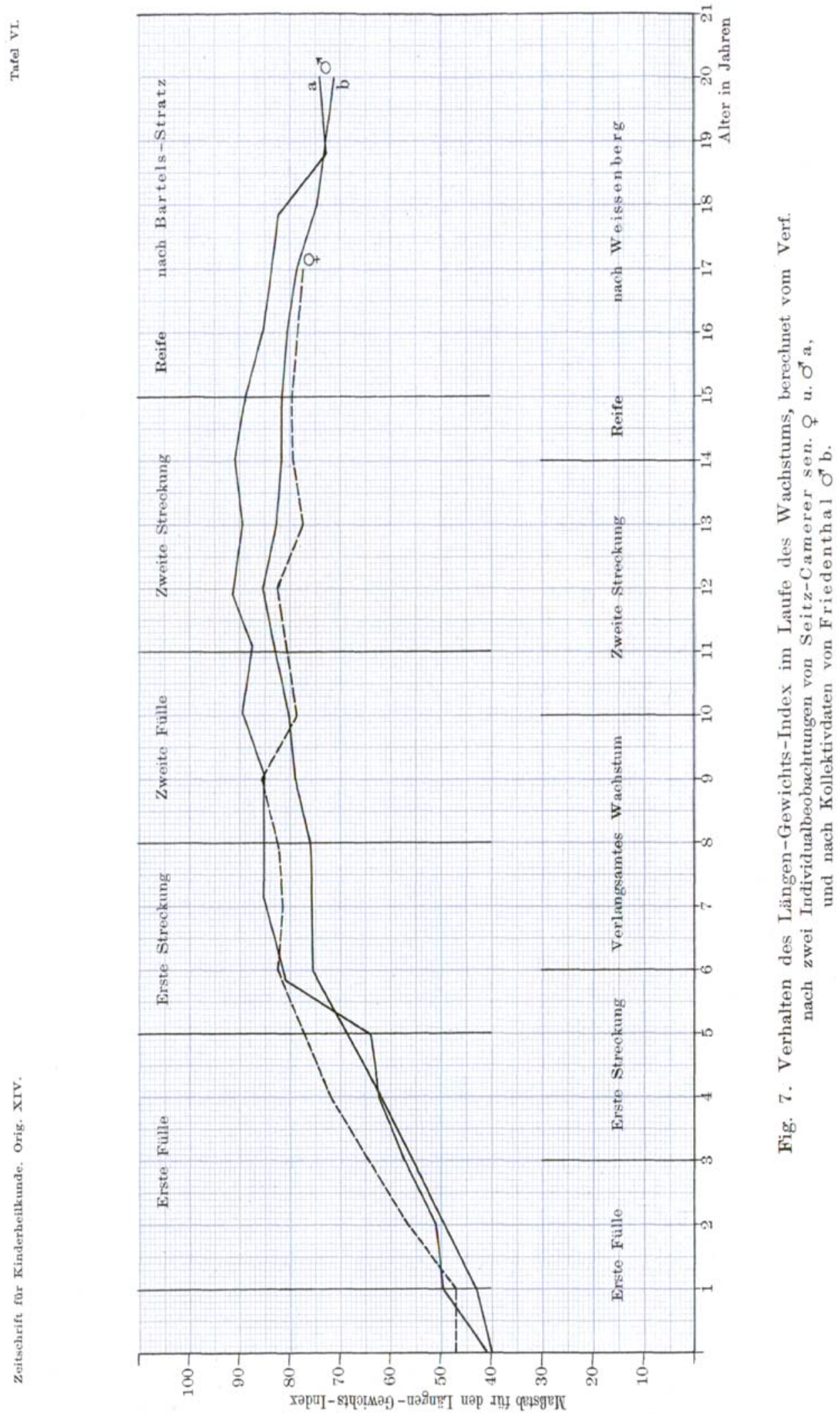




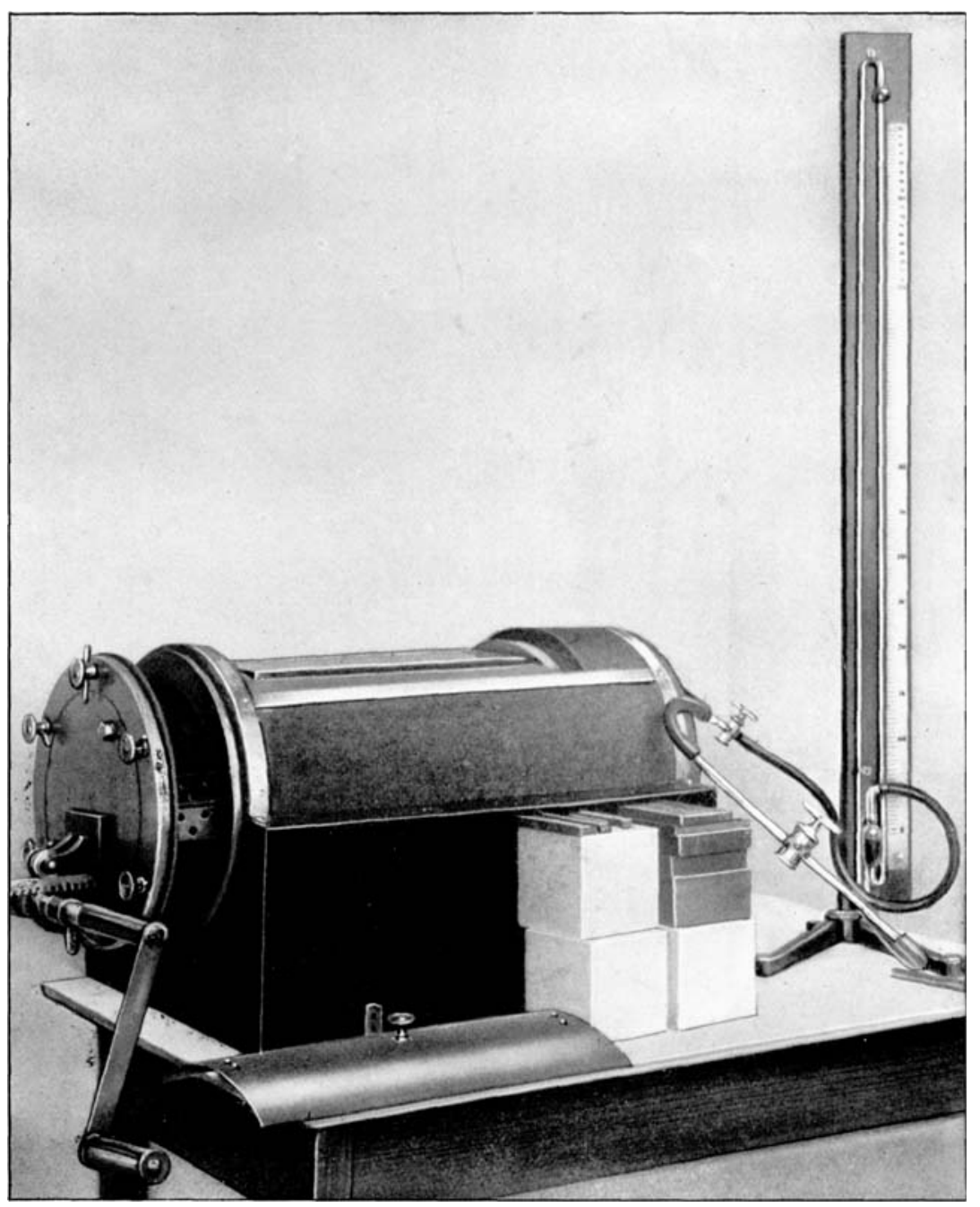

Fig. 11. 


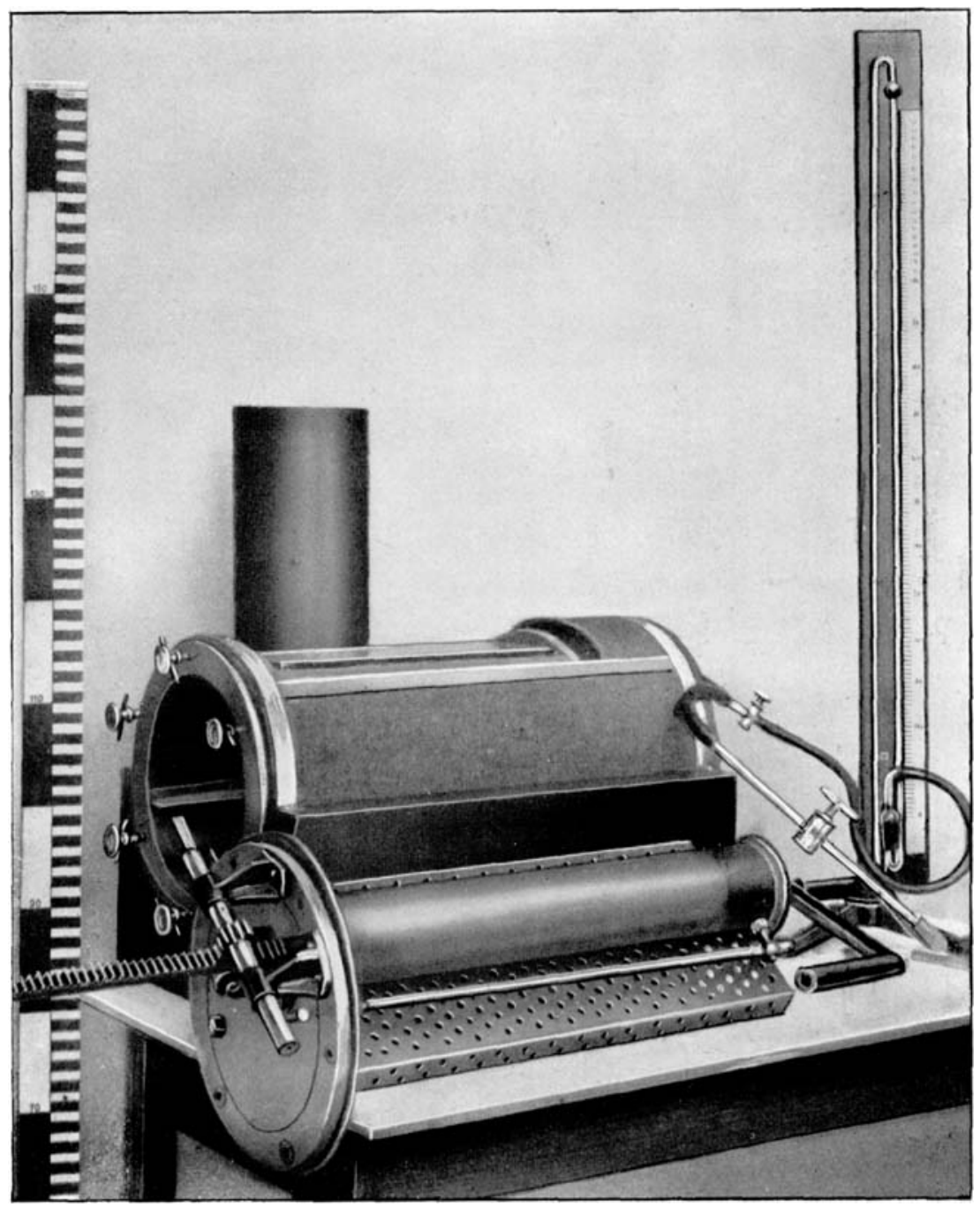

Fig. 12.

Pfaundler, Körpermaß-Studien. 